\title{
Evaluation of initial methadone dosing for prevention of iatrogenic opioid abstinence syndrome in children
}

\author{
Peter N. Johnson*, Jamie Miller and Donald Harrison \\ Department of Pharmacy, Clinical and Administrative Sciences, University of Oklahoma, College of Pharmacy, \\ Oklahoma City, OK, USA
}

\author{
Received 23 November 2010 \\ Revised 25 March 2011 \\ Accepted 27 March 2011
}

\begin{abstract}
Methadone is commonly prescribed for prevention of iatrogenic opioid abstinence syndrome (IOAS) in critically ill children. However, there is a paucity of data on the recommended initial dosage requirements. Data was retrospectively collected from January-December 31, 2008 and included demographics, methadone regimen, and IOAS symptoms. The primary objective was to determine the initial methadone dosing utilized (i.e., $\mathrm{mg} / \mathrm{kg} / \mathrm{day}$ ) and the impact that this had on the number of dosage changes required for prevention of IOAS in patients $<18 \mathrm{yr}$ within $72 \mathrm{hr}$ following discontinuation of opioid continuous infusion (CI). Secondary objectives included a comparison of methadone dose changes within $72 \mathrm{hr}$ of intravenously opioid discontinuation and opioid CI requirements based on initial doses above and below the median level (i.e., $\mathrm{mg} / \mathrm{kg} / \mathrm{day}$ ). Between-group analysis was performed using descriptive and inferential statistics. A step-wise regression was employed to assess relationships between the initial dose ( $\mathrm{mg} / \mathrm{kg} / \mathrm{day})$ and several independent variables. Fifty-five patients were included for analysis. The median initial dose was $0.84 \mathrm{mg} / \mathrm{kg} / \mathrm{day}$. The "low-dose" group included patients receiving $<0.84 \mathrm{mg} / \mathrm{kg} / \mathrm{day}$ ( $\mathrm{n}=27$ ); the "high-dose" group included patients receiving $\geq 0.84 \mathrm{mg} / \mathrm{kg} / \mathrm{day}(\mathrm{n}=28)$. The majority of patients $(81.8 \%)$ received doses every $6 \mathrm{hr}$. Twenty-three patients $(41.8 \%)$ developed IOAS and required an increase in dose while six patients $(10.9 \%)$ required a decrease. No significant differences were found between groups. The cumulative and peak fentanyl CI doses were the only significant predictors of total $\mathrm{mg} / \mathrm{kg} / \mathrm{day}$ dose of methadone $(P<0.01)$. The initial methadone dosage regimens varied greatly in our patients. Approximately half required a change in their methadone dose. Based on our findings and a review of the literature, there is not a one-size fits all approach for determination of the initial methadone dose. Adoption of a consistent monitoring tool should be utilized for all children across the continuum of care to prevent IOAS and the oversedation noted with excessive methadone dosing.
\end{abstract}

Keywords: Children, methadone, opioid withdrawal

\section{Introduction}

Sedation and analgesia are important considerations for children admitted to the pediatric intensive

*Corresponding author: Peter N. Johnson, Department of Pharmacy, Clinical and Administrative Sciences, University of Oklahoma College of Pharmacy, 1110 N. Stonewall Ave., CPB 206, 73117, Oklahoma City, OK, USA. Tel.: +1 405271 2730; Fax: +1 405271 5424; E-mail: peter-johnson@ouhsc.edu. care unit (PICU). A number of sedative/analgesic regimens are used including continuous infusion (CI) of opioids, benzodiazepines, ketamine, and dexmedetomidine. In a recent survey of PICU fellowship program directors regarding sedative/analgesic practices by Twite et al. [1], the majority of responders reported that the two most common agents used as part of sedative/analgesic regimens were fentanyl and morphine. 
Prolonged opioid exposure has been associated with iatrogenic opioid abstinence syndrome (IOAS) following discontinuation. The incidence of IOAS in neonates and children has been estimated between $35-57 \%$ [2-4]. Several risk factors for IOAS have been identified and include extended opioid duration (> 5-7 days), elevated total cumulative doses, rapid tapering, and/or abrupt discontinuation [2,3]. IOAS symptoms include central nervous system irritability (e.g., anxiety, agitation, grimacing, sleep disturbance), gastrointestinal dysfunction (e.g., vomiting, diarrhea), and autonomic dysfunction (e.g., tachypnea, diaphoresis, and hypertension) [5]. These symptoms could be associated with increased morbidity, prolonged hospital stay, and patient discomfort [4].

There are a number of interventions that have been utilized for opioid withdrawal including methadone, alpha-2 agonists, and transdermal fentanyl, but there is not one accepted therapy for all patients [6-8]. Methadone, a long-acting opioid agonist with $\mathrm{N}$-methyl-D-aspartate (NMDA) antagonist effects, is a commonly used agent, and researchers have published reports of methadone dosing strategies for prevention of IOAS [9-17]. These strategies can be generally classified as a formula-based or dosing-based protocol. For a specific patient, the initial methadone dose selected would differ based on the specific protocol utilized. Despite the use of these published protocols, some patients have still required dosage adjustments for IOAS symptoms. As a result, there is no standard consensus on the initial methadone dose utilized to prevent or treat IOAS in infants/children. Over the last several years, prescribers in our institution have used various initial methadones dosing for prevention of IOAS. The objective of this study was to determine the initial methadone dosing utilized (i.e., $\mathrm{mg} / \mathrm{kg} /$ day) and the impact that this had on the number of dosage changes required within $72 \mathrm{hr}$ following discontinuation of opioid CI.

\section{Materials and methods}

\subsection{Study design}

This was a descriptive, retrospective study of patients $<18$ yr of age from January 1, to December 31, 2008 who received enteral methadone for prevention of IOAS. The study was conducted in a tertiary care academic hospital licensed for 230-beds, including 25PICU beds and 88-neonatal intensive care unit (ICU) inpatient beds. Following Institutional Review Board approval, patients were identified through the institution's electronic database, Meditech (Medical Information Technology, Inc., Westwood, MA). Patients were excluded if they received methadone for treatment of IOAS; this was defined as IOAS symptoms prompting initiation of methadone. Other exclusion factors included initiation of methadone for treatment of chronic pain/ palliative care and treatment of neonatal abstinence syndrome. In addition, patients with incomplete medical records were excluded from data analysis.

\subsection{Study objectives and data collection}

Data were collected by two investigators (PNJ and JLM). For each subject, a number of baseline data were collected, including age at initiation of methadone, gender, hospital and ICU length of stay, and diagnosis at the time of admission (e.g., post-operative surgery/trauma, septic shock/infection, pulmonary disease, other). Each patient's regimen for sedation and analgesia were recorded in an effort to identify what agents were received and to quantify opioid exposure. Specific data were collected regarding the fentanyl CI regimen including cumulative dose of fentanyl $(\mu \mathrm{g} / \mathrm{kg})$, peak rate of fentanyl $(\mu \mathrm{g} / \mathrm{kg} / \mathrm{hr})$, duration (days), and dose ( $\mu \mathrm{g} / \mathrm{kg} /$ hr) immediately prior to fentanyl discontinuation. In addition, the duration of overlap was also collected; for the purpose of this study, this was defined as the number of hours that the patient received the fentanyl CI and methadone. In our institution, fentanyl is the most common opioid analgesic agent prescribed for sedation/analgesia. Therefore, for patients who may have received other opioid agents, their dosage regimen was converted to fentanyl and recorded (i.e., $10 \mathrm{mg}$ morphine given intramuscularly/intravenous $[\mathrm{IV}]=0.1 \mathrm{mg}$ of fentanyl given intramuscularly/IV). In addition, data were collected for the initial methadone dosage regimen including initial dose $(\mathrm{mg} / \mathrm{kg} / \mathrm{dose}$ and $\mathrm{mg} / \mathrm{kg} /$ day $)$, initial dosing interval, and requirement for adjustment of initial dose (e.g., increased, decreased, no change). Concomitant use of an enteral diazepam taper for prevention of withdrawal following prolonged use of benzodiazepine CI was also noted.

Data were also collected to assess development of IOAS. The physicians' and nursing's progress notes were reviewed for symptoms of IOAS recorded within $72 \mathrm{hr}$ following the last dosage reduction/discontinuation of the fentanyl CI [9]. Symptoms that were evaluated included dilated pupils, diarrhea, gastrointestinal problems (i.e., regurgitation, emesis), increased muscle tone, 
seizures, tremors, inconsolable crying, agitation/irritability, tachycardia (i.e., children $<1$ yr of age-unexplained heart rate $>40$ beats $/ \mathrm{min}$ from baseline for $10 \mathrm{~min}$ $>1 \mathrm{yr}$ of age-unexplained heart rate $>30$ beats/min from baseline for $10 \mathrm{~min}$ ), and hypertension (unexplained systolic BP > $30 \mathrm{mmHg}$ from baseline for $10 \mathrm{~min}$ ) [10]. These symptoms must have been noted by the medical team and required intervention (e.g., defined as greater than one dose of a breakthrough opioid agent given to alleviate IOAS symptoms in a $24 \mathrm{hr}$ period and/or a dosage increase in methadone) [9].

The primary objective was to identify the initial methadone dosage regimen (i.e., $\mathrm{mg} / \mathrm{kg} /$ day) and the requirement of dosage adjustments (e.g., increase or decrease) within $72 \mathrm{hr}$ following discontinuation of opioid CI. Due to heterogeneity in initial dosing requirements, patients were arbitrarily sorted into "high" and "low" dose methadone groups using the median initial methadone dose (i.e., $\mathrm{mg} / \mathrm{kg} /$ day). Secondary objectives included a comparison between groups of the fentanyl CI dosage requirements and types of concomitant sedative agents required. Additionally, the number of dosage adjustments was compared between groups. An attempt was also made to compare the number of patients requiring an enteral diazepam taper between groups.

\subsection{Statistical analysis}

Data were summarized using the mean, median, and range for continuous data and percentages for categorical data where appropriate. The arbitrary division (i.e., median initial dose in $\mathrm{mg} / \mathrm{kg} / \mathrm{day}$ ) was selected to maximize the statistical power to compare baseline demographics, IOAS symptoms, concomitant sedation/analgesic agents, and methadone dosage frequency/duration between the low-dose and highdose groups. Descriptive and inferential statistics were performed using paired $t$-tests, Fischer's exact test, or Chi-square test when appropriate.

A linear regression analysis was employed to assess relationships between initial methadone dose $(\mathrm{mg} / \mathrm{kg} /$ day) and selected independent variables including cumulative fentanyl CI dose, peak fentanyl CI dose, duration of fentanyl CI, dose prior to fentanyl CI discontinuation, and duration of overlap. A post hoc, logistic regression was conducted to ascertain the association of IOAS with several independent variables including duration of fentanyl CI, cumulative fentanyl CI dose, peak fentanyl $\mathrm{Cl}$ dose, dose prior to fentanyl CI discontinuation, and duration of overlap. All data management and statistics were performed using Stata 10 [18]. A $P$ value $<0.05$ was considered statistically significant.

\section{Results}

\subsection{Patient demographics}

Ninety-six patients were identified (Fig. 1). Reasons for patient exclusion included chronic pain/palliative care $(n=11)$, neonatal abstinence syndrome $(n=3)$, treatment of withdrawal $(\mathrm{n}=11)$, incomplete records $(n=9)$, or other $(n=7)$. Fifty-five were included in this study with a median age of $0.5 \mathrm{yr}$ (range 0.03-12.2) with a statistically significant difference in the median age between the low-and high-dose groups, 0.3 versus 1.1 yr respectively $(P=0.01)$ (Table 1$)$. Of those patients categorized as neonates (i.e., less than 30 days old) $(n=7)$, all but one of these infants were full-term and received methadone to facilitate fentanyl discontinuation and prevent IOAS. Methadone was initiated in one premature neonate (i.e., 24 wk-gestation) at a postmenstrual age of 33 wk-gestation.

The median initial methadone dose per day was 0.84 $\mathrm{mg} / \mathrm{kg} /$ day. The "low-dose" group included patients with a dose $<0.84 \mathrm{mg} / \mathrm{kg} /$ day $(\mathrm{n}=27)$; the "high-dose" group included patients with a dose $\geq 0.84 \mathrm{mg} / \mathrm{kg} / \mathrm{day}$ $(n=28)$. Table 1 includes the baseline demographics between the "high" and "low" methadone groups. The only significant differences between groups were age at the time of admission and weight.

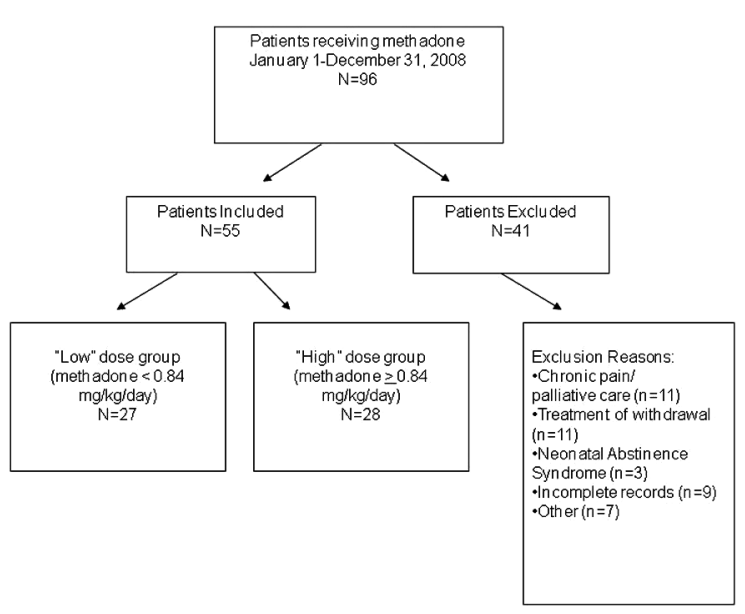

Fig. 1. Sample flowchart. 
Table 1

Baseline demographics $(\mathrm{n}=55)$

\begin{tabular}{|c|c|c|c|}
\hline \multirow[t]{2}{*}{ Characteristics } & $\begin{array}{c}\text { Low dose group } \\
(<0.84 \mathrm{mg} / \mathrm{kg} / \text { day })(\mathrm{n}=27)\end{array}$ & $\begin{array}{c}\text { High dose group } \\
(\geq 0.84 \mathrm{mg} / \mathrm{kg} / \text { day })(\mathrm{n}=28)\end{array}$ & \multirow[t]{2}{*}{$P$} \\
\hline & \multicolumn{2}{|c|}{$\mathrm{n}(\%)$} & \\
\hline Male & $18(66.7)$ & $17(60.7)$ & 0.20 \\
\hline \multicolumn{4}{|l|}{ Admitting diagnosis } \\
\hline Post-operative/trauma & $9(33.3)$ & $12(42.9)$ & 0.17 \\
\hline Infection/septic shock & $4(14.8)$ & $4(14.3)$ & 0.30 \\
\hline Pulmonary disease & $12(44.4)$ & $10(35.7)$ & 0.18 \\
\hline Other & $2(7.4)$ & $2(7.1)$ & 0.39 \\
\hline \multicolumn{4}{|c|}{ Median (range) } \\
\hline Age $(y r)$ & $0.3(0.03-12.2)$ & $1.1(0.05-8.1)$ & 0.01 \\
\hline Weight (kg) & $5.0(2.2-30)$ & $8.7(2.8-51.4)$ & 0.01 \\
\hline \multicolumn{4}{|l|}{ Length of stay (day) } \\
\hline $\begin{array}{l}\text { Intensive care unit } \\
\text { length of stay }\end{array}$ & $15(7-158)$ & $20(8-155)$ & 0.90 \\
\hline Total length of stay & $30(7-158)$ & $33.5(9-167)$ & 0.22 \\
\hline
\end{tabular}

Table 2

Fentanyl continuous infusion regimen

\begin{tabular}{lccc}
\hline Characteristics & $\begin{array}{c}\text { Low dose group } \\
(<0.84 \mathrm{mg} / \mathrm{kg} / \mathrm{day}) \\
(\mathrm{n}=27)\end{array}$ & $\begin{array}{c}\text { High dose group } \\
(\geq 0.84 \mathrm{mg} / \mathrm{kg} / \mathrm{day}) \\
(\mathrm{n}=28)\end{array}$ \\
\cline { 2 - 3 } & \multicolumn{2}{c}{ Mean $\pm \mathrm{SD}$} \\
\hline Cumulative dose $(\mu \mathrm{g} / \mathrm{kg})$ & $516.6 \pm 301.4$ & $1752 \pm 1365.8$ & \\
Peak rate of administration $(\mu \mathrm{g} / \mathrm{kg} / \mathrm{hr})$ & $3.6 \pm 1.9$ & $7.2 \pm 4.0$ & $<0.01$ \\
Duration $(\mathrm{hr})$ & $252.5 \pm 172.8$ & $393.8 \pm 331.4$ & $<.01$ \\
Dose prior to discontinuation $(\mu \mathrm{g} / \mathrm{hr}) *$ & $1.9 \pm 3.1$ & $2.3 \pm 2.1$ & 0.04 \\
Duration of overlap $(\mathrm{hr}) * *$ & $33.4 \pm 37.7$ & $67.2 \pm 91.5$ & 0.06 \\
\hline *Dose administered just prior to discontinuation of fentanyl continuous infusion. \\
**Number of hours that the patient received both the opioid continuous infusion and methadone prior to discontinuation of the opioid \\
continuous infusion.
\end{tabular}

\subsection{Sedative and analgesic exposure}

Table 2 includes the data for fentanyl CI. Overall, the patients in the "low-dose" group had significantly less exposure (i.e., peak rate and duration) to opioids than the "high dose" group. There was a statistically significant difference in the mean cumulative fentanyl CI dose between the low- and high-dose groups, 516.6 versus $1752 \mu \mathrm{g} / \mathrm{kg}$ respectively $(P<0.01)$. However, there was no significant difference in the mean dose of the fentanyl CI prior to discontinuation between the "lowdose" and "high-dose" groups, 1.9 versus $2.3 \mu \mathrm{g} / \mathrm{kg} / \mathrm{hr}$, $P=0.40$. In addition, there was no significant difference in the duration of overlap between groups.

The sedative and analgesic regimens of this cohort are included in Fig. 2. Despite the relatively small number of patients included in this study, there were a number of different regimens utilized. The most common regimen for both groups included the combination of a fentanyl CI and a benzodiazepine CI (e.g., midazolam). It is important to note that patients received anywhere from one to four agents for sedation and analgesia at any one time during their ICU stay. However, due to the heterogeneity in the patient population, statistical analysis was not conducted between groups.

\subsection{Initial methadone dosage regimen}

A description of the initial methadone regimens is found in table 3 . Though not surprising, there was a statistically significant difference in the initial methadone dose between groups. For a majority of patients, the initial methadone dosage frequency was every $6 \mathrm{hr}$. 


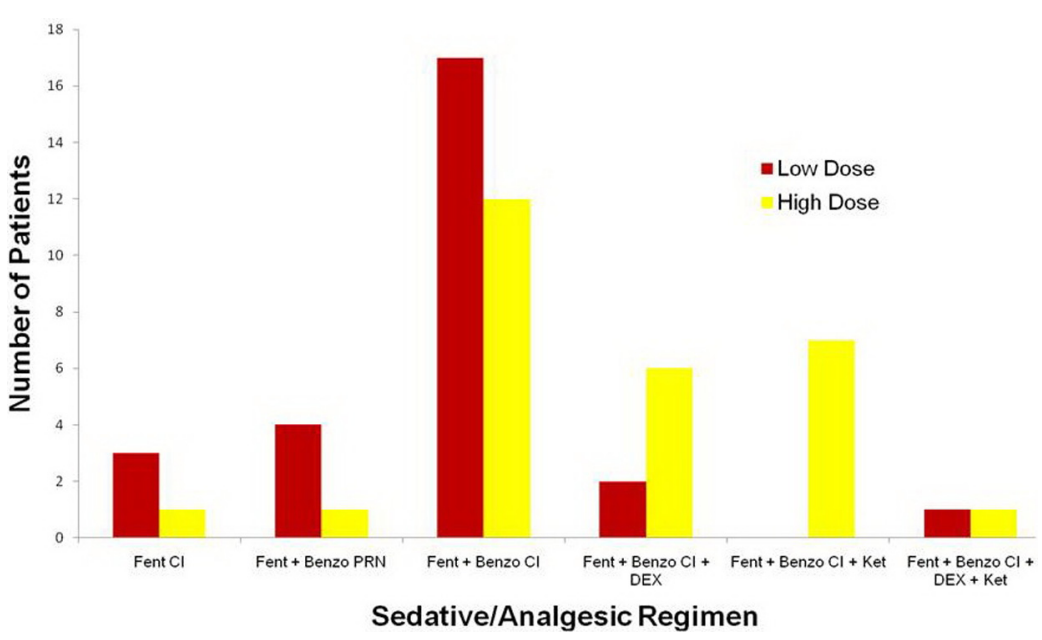

Fig. 2. Sedative/analgesic regimens.

Table 3

Initial methadone regimen $(\mathrm{n}=55)$

\begin{tabular}{|c|c|c|c|}
\hline \multirow[t]{2}{*}{ Characteristics } & $\begin{array}{c}\text { Low dose group } \\
(<0.84 \mathrm{mg} / \mathrm{kg} / \text { day })(\mathrm{n}=27)\end{array}$ & $\begin{array}{c}\text { High dose group } \\
(\geq 0.84 \mathrm{mg} / \mathrm{kg} / \text { day })(\mathrm{n}=28)\end{array}$ & \multirow[t]{2}{*}{$P$} \\
\hline & \multicolumn{2}{|c|}{ Mean \pm SD } & \\
\hline \multicolumn{4}{|l|}{ Dose } \\
\hline $\mathrm{mg} / \mathrm{kg} /$ dose & $0.1 \pm 0.04$ & $0.4 \pm 0.3$ & $<0.01$ \\
\hline $\mathrm{mg} / \mathrm{kg} /$ day & $0.5 \pm 0.2$ & $1.8 \pm 1.0$ & $<0.01$ \\
\hline \multicolumn{4}{|c|}{ n $(\%)$} \\
\hline \multicolumn{4}{|l|}{ Initial dosing frequency } \\
\hline Q $12 \mathrm{hr}$ & $1(3.7)$ & $0(0)$ & 0.49 \\
\hline Q $8 \mathrm{hr}$ & $8(29.6)$ & $1(3.6)$ & $<0.01$ \\
\hline Q $6 \mathrm{hr}$ & $18(66.7)$ & $27(96.4)$ & $<0.01$ \\
\hline \multicolumn{4}{|l|}{ Dosage change } \\
\hline None & $14(51.9)$ & $12(42.9)$ & 0.17 \\
\hline Increase & $9(33.3)$ & $14(50.0)$ & 0.17 \\
\hline Decrease & $4(14.8)$ & $2(7.1)$ & 0.23 \\
\hline $\begin{array}{l}\text { Developed iatrogenic opioid } \\
\text { abstinence syndrome }\end{array}$ & $11(40.7)$ & $12(42.9)$ & 0.21 \\
\hline
\end{tabular}

Twenty-nine patients $(52.7 \%)$ required either a decrease or increase in dose based on their clinical symptoms. Twenty-three of these children $(79.3 \%)$ required a dosage increase to control their signs and symptoms of IOAS. For the patients who required a decrease in methadone dose, the most common reason was for over-sedation. There was no significant difference in the number of children requiring a dosage change between groups.

Thirty-three patients $(60 \%)$ received other agents in combination with initiation of methadone. Thirty-two patients $(58.1 \%)$ received enteral diazepam for prevention of benzodiazepine withdrawal. There was a significant difference in the number of patients receiving diazepam tapers between the low and high methadone dose groups, 12 versus $20, P=0.03$, respectively. Although the specific tapering schedule with diazepam was not recorded, consistently, the doses of methadone and diazepam were reduced on an alternating schedule. Of note, one patient in the "high-dose" group received three days of enteral clonidine in combination with methadone for prevention of drug withdrawal; after this time, the clonidine was discontinued. Another patient in the "high-dose" group received four days of transdermal fentanyl in addition to methadone and diazepam. After this period, the transdermal fentanyl was discontinued, and the patient continued on a methadone and diazepam taper. 
Although not a specific focus of this study, the duration of the methadone taper (i.e., duration of in-patient and outpatient days of therapy) was collected in fortyeight patients. Seven patients were not included for analysis because they were discharged to a long-term care facility without specific recommendations for tapering $(n=4)$, had incomplete records $(n=2)$, or expired $(n=1)$. There was a statistically significant difference in the mean duration of the methadone taper between the low-dose $(\mathrm{n}=23)$ and high-dose $(\mathrm{n}=$ 25 ) groups, $14.7 \pm 9.2$ versus $26.2 \pm 11.5$ respectively $(P<0.01)$. Most patients had dose reductions of approximately $10-20 \%$ of the initial dose every $24-48 \mathrm{hr}$. The reduction in dose and the interval at which the doses were changed was prescriber dependent.

\subsection{Regression analysis}

A linear regression analysis was conducted (Table 4). In this analysis, the dependent variable was the total $\mathrm{mg} /$ $\mathrm{kg}$ /day dose of methadone. Because of the wide interpatient variability, several factors were included as independent variables in the analysis to determine if any of these factors were predictive of the initial starting dose. The only significant predictors in this analysis were the cumulative and peak fentanyl $\mathrm{CI}$ dose $(P<0.01)$. In the post hoc logistic regression, the presence of IOAS symptoms was the dependent variable. None of the independent variables (i.e., duration of fentanyl CI, cumulative fentanyl CI dose, peak fentanyl $\mathrm{Cl}$ dose, dose prior to fentanyl CI discontinuation, and duration of overlap) were significant predicators of development of symptoms of IOAS in this cohort.

Table 4

Regression analysis

\begin{tabular}{lcc}
\hline Variable & $\begin{array}{c}\text { Is the variable a } \\
\text { predictor of total } \\
\text { mg/kg/day required }\end{array}$ & $P$ \\
\hline Cumulative fentanyl CI dose & Yes & $<0.01$ \\
Peak fentanyl CI dose & Yes & $<0.01$ \\
Duration of fentanyl CI & No & 0.99 \\
Fentanyl CI dose prior to & No & 0.48 \\
$\quad$ discontinuation* & No & 0.56 \\
$\begin{array}{l}\text { Duration of overlap** } \\
\text { CI }=\text { Continuous infusion. }\end{array}$ & \\
*Dose administered just prior to discontinuation of fentanyl continuous \\
infusion. \\
$* *$ Number of hours that the patient received both the opioid continuous \\
infusion and methadone prior to discontinuation of the opioid continuous \\
infusion.
\end{tabular}

\section{Discussion}

This was a retrospective, descriptive study evaluating the initial methadone dosing and the impact that the initial dose selected had on the number of dose changes required in critically ill children for prevention of IOAS. Many experts have suggested and/or studied various dosing protocols [6,9-16,19]. Currently in our institution, a standard dosing protocol has not been utilized. Therefore, the initial dose selection depends upon clinician discretion, and we did not find an identifiable pattern with regards to initial dose selection. Our cohort had a median initial dose of $0.84 \mathrm{mg} / \mathrm{kg} /$ day that was administered in the majority of patients every $6 \mathrm{hr}$. Many of the previous research reports have not provided specific details on the number of dosage increases and decreases required following selection of the initial dose. In some of the studies, it is hard to extrapolate the dosing frequency and $\mathrm{mg} /$ $\mathrm{kg}$ dosing required, as these variables are not specifically reported. As a result, it can be difficult to apply these results to clinical practice without this specific information. In comparison to previous studies, we also set out to report the number of dosage increases and decreases required following initiation of methadone and to report the complete initial regimen (i.e., $\mathrm{mg} / \mathrm{dose}, \mathrm{mg} / \mathrm{kg} / \mathrm{dose}$, and dosing interval).

There are two general approaches to methadone dosing available from the literature (i.e., formulabased and dosing-based protocols). One case report, review article, and small retrospective study discuss strategies for initial methadone dosing based on an empiric dosage-protocol $[9,15,20]$. The initial dose from these three reports ranges from $0.05-0.1 \mathrm{mg} / \mathrm{kg} /$ dose every six or twelve hours. In a retrospective study by Lugo et al. [9], the investigators evaluated twentytwo children who received a fentanyl CI or equivalent opioid for $\geq 9$ days and a median cumulative fentanyl CI dose of $1302 \mu \mathrm{g} / \mathrm{kg}$ (range 354-7535). All of the patients received an initial methadone dose of $0.1 \mathrm{mg} / \mathrm{kg} / \mathrm{dose}$ every $6 \mathrm{hr}$ until their fentanyl CI was discontinued. Their protocol had a strict tapering schedule for the fentanyl CI with a $10-15 \%$ decrease in infusion rate every $8 \mathrm{hr}$. The authors found that one patient had documented IOAS requiring an extended fentanyl CI taper and an increase in their methadone dose. Their mean initial methadone starting dose was $0.5 \pm 0.22 \mathrm{mg} / \mathrm{kg} /$ day reflecting the fact that seven patients required an empiric dosage adjustment due to a significantly higher cumulative fentanyl CI dose than the other patients. The authors concluded that this 
dosing protocol may work for most patients but recognized that patients with higher cumulative fentanyl CI doses may require a higher dose to prevent IOAS.

Four formula-based approaches for methadone dosing have been described in the literature and have been formally evaluated [10-14,16]. In a case report, Tobias recommended a formula-based approach where the initial methadone dose was equivalent to total daily fentanyl CI dose in mg/day administered every $12 \mathrm{hr}$; however, a formal evaluation was not conducted [14]. A later retrospective study described the results from this approach in 18 children [16]. In two different prospective studies by Meyer and Berens [11] and Berens et al. [12], the authors described the use of a formula approach in 66 children where their initial methadone dose equaled their fentanyl CI dose $(\mu \mathrm{g} / \mathrm{kg} / \mathrm{hr}) \times 60$ $\min \times 2$, administered every twelve hours. Whereas, Robertson et al. [13] reported the results in a prospective study of ten children in the formula-based protocol group who received an initial methadone dose that equaled their hourly morphine or fentanyl CI dose based on an equipotent dose conversion to methadone administered every $6 \mathrm{hr}$ [13]. Siddappa et al. [10] retrospectively evaluated the use of a methadone formula-based protocol in 30 children. Each patient's methadone dose was determined by calculating the fentanyl CI daily dose $(\mu \mathrm{g} / \mathrm{kg}) \times 3$, administered every $8 \mathrm{hr}$. In the studies by Siddappa et al. [10] and Robertson et al. [13], methadone was initiated via the IV route on day 1 and changed to the enteral route on day 2 or 3 , with no dose adjustments when converting from the IV to enteral route. All four reports describe different methods for calculation of an initial methadone dose.

In our current study, we identified the median initial methadone dose per day and arbitrarily chose this dose as the dividing point to establish patients above and below the median dose per day. Between these two groups, we compared the number of patients who developed IOAS and patients who required subsequent changes in dose. Unlike some of the formula-based approaches, the majority of patients received enteral methadone administered every $6 \mathrm{hr}$. Similar to the results by Lugo et al. [9], the majority of patients in the high-dose group received a median cumulative fentanyl CI $1442 \mu \mathrm{g} /$ $\mathrm{kg}$ with greater than nine days of fentanyl exposure. However, the initial methadone dose in their cohort was $0.5 \mathrm{mg} / \mathrm{kg} /$ day where as our cohort were initiated on significantly higher median dose of $0.84 \mathrm{mg} / \mathrm{kg} / \mathrm{day}$. Our initial dose is difficult to compare to the published protocols as none of the reports describe the actual initial methadone dose per day or dose per kilogram.

We noted a relatively high incidence of IOAS in our patient population compared to the published studies describing the utilization of methadone for prevention of withdrawal. Based on our evaluation, twenty-three children (41.8\%) exhibited symptoms and were treated for acute opioid withdrawal. In the published studies evaluating both formula-based and protocol-based approaches, the rate of IOAS following initiation of methadone for prevention of withdrawal was approximately 4.5-30\% [9-13,16]. Patients with IOAS in these previously mentioned studies received an increase in methadone dose or administration of a short-acting opioid for acute symptoms; however, the authors report that in some cases the withdrawal episodes were not clinically significant and required no additional interventions. In contrast to these studies, we only designated patients with IOAS if they had both symptoms and received additional interventions to alleviate their symptoms (e.g., increase in methadone dose, administration of shortacting opioid). It is difficult to determine why our incidence of IOAS is higher than previous reports. There a multitude of factors, that may have influenced this finding in our cohort. Because there is no one standardized approach utilized in the patients in our study, some patients may have had too low of an initial methadone dose in relation to their total fentanyl exposure or had inconsistencies in the way that their fentanyl CI was tapered off and overlapped with methadone. Taking these factors in consideration, we performed an ad-hoc logistic regression with IOAS as the dependent variable but did not find any significant predictors of withdrawal in the model.

Six patients $(10.9 \%)$ required a decrease in dose, but there were no significant differences between groups. Based on our review of the previous studies, only one study reported the need to decrease the initial methadone dose based on the patient's clinical status (e.g., over-sedation) [16]. It is difficult to identify if this finding is directly related to the methadone dose itself or from other factors associated with the clinical status of these patients due to their critical-illness.

Over half of our population was initiated on enteral diazepam for prevention of benzodiazepine withdrawal. Three published reports have described withdrawal symptoms in children following abrupt discontinuation of benzodiazepine CI [21-23]. Based on these reports, the overall incidence of withdrawal is estimated at $17-24 \%$ which is lower than that reported for opioid 
withdrawal $[21,22]$. A potential confounding factor in this study is that benzodiazepine withdrawal symptoms could have been mistaken for opioid withdrawal. However, based on our strict criteria for defining IOAS, all patients had to exhibit documented symptoms of IOAS and required an intervention (e.g., breakthrough opioid or increase methadone dose). In review of the medical records, all patients appeared to respond to this intervention based on the clinician assessment. In addition, in this patient cohort, enteral diazepam and methadone were tapered on alternating days allowing the clinicians to observe the effects of a decreased dose in one agent versus the other.

\subsection{Limitations}

There are several limitations to our study that must be considered. The data in this study was collected retrospectively, and no one standardized evaluation tool was utilized to assess for IOAS. However, we used a very strict definition of IOAS that included both specific symptoms and requirements for intervention. As previously mentioned, we do not currently have a standardized approach to determine the initial methadone dose. This made an assessment of outcomes difficult to interpret. Thus, we arbitrarily chose the median initial methadone dose per day to maximize statistical power to compare outcomes between groups. It is not clear if selection of a different breakpoint would have resulted in significant differences in IOAS and number of patients requiring dosage changes. However, the post-hoc regression analysis suggested no specific relationship between the methadone dose and development of IOAS. Our final limitation is that this was a small study in a single academic medical center, but our study size was comparable to the other published reports.

\subsection{Implications}

Based on a review of the literature and the results of this study, there are several take-home points to be noted. First, a specific monitoring tool to assess for opioid withdrawal should be utilized. Second, clinicians should adopt one specific protocol at their institution to select the initial methadone dose. A consensus among prescribers should utilized to ensure that children are assessed with the same monitoring tool and dosed according to the same protocol regardless of the patient's age and across the continuum of care (i.e., ICU versus general wards). By initiating these efforts, children with prolonged opioid exposure may have diminished morbidities associated with IOAS including general discomfort and hospital stay.

In regards to the selection of the type of protocol that should be adopted, it is our opinion that dosing-based protocols would be preferred over formula-based protocols in critically ill children for the following reasons. First, four formula-based protocols have been described; all four methods have very different approaches with varying success. Next, these formula-based protocols utilize the equipotent dose from IV opioids to methadone based on half-lives based on population averages. Due to the likelihood of altered renal and hepatic function in critical illness, these population averages may underestimate the true half-life of these agents. For example, different half-lives of fentanyl CI were found in perioperative children versus critically-ill children [24,25]. If these differences were not assessed, then the calculated dose would exceed the dose needed and would prolong the duration of the opioid taper. Because analgesia is not the goal in this case, it seems prudent that the lowest dose possible should be utilized to prevent physiologic withdrawal symptoms rather the equipotent analgesic dose. One reasonable approach for patients with low-risk factors (i.e., cumulative fentanyl CI dose $\geq 1.5$ and $<2.5 \mathrm{mg} / \mathrm{kg}$ and duration of fentanyl $\mathrm{CI} \geq 5$ and $<9$ days) is to initiate methadone at $0.1 \mathrm{mg} /$ $\mathrm{kg} /$ dose every $6 \mathrm{hr}$. For patients with high-risk factors (i.e., cumulative fentanyl CI dose $\geq 2.5 \mathrm{mg} / \mathrm{kg}$ and duration of fentanyl $\mathrm{CI} \geq 9$ days), methadone can be initiated $0.2-0.4 \mathrm{mg} / \mathrm{kg} /$ dose every $6 \mathrm{hr}$. Clinicians should be mindful that regardless of the initial dose utilized that patients may require more or less drug based on their individual pharmacokinetic variables, drug interactions, and/or drug-disease interactions. This is evidenced by the fact that despite the use of previously published protocols, up to a third of patients still exhibited signs of IOAS. Thus, there is not a one-size fits all approach that will work for all patients.

In conclusion, this study describes the initial dose selection of methadone in one institution without a current standardized approach. The median dose identified in this cohort was $0.84 \mathrm{mg} / \mathrm{kg} / \mathrm{day}$ most often divided every $6 \mathrm{hr}$. Approximately $40 \%$ developed IOAS and $10 \%$ required a decrease in dose with no differences noted between those above and below the median dose per day. Based on our findings and a review of published literature, there is not a one-size 
fits all approach for determination of the initial methadone dose. Adoption of a consistent monitoring tool should be utilized for all children across the continuum of care to prevent IOAS and the over-sedation noted with excessive methadone dosing.

\section{References}

[1] Twite MD, Rashid A, Zuk J, Friesen RH. Sedation, analgesia, and neuromuscular blockade in the pediatric intensive care unit: survey of fellowship training programs. Pediatr Crit Care Med 2004;5(6):521-32.

[2] Katz R, Kelly HW, Hsi A. Prospective study on the occurrence of withdrawal in critically ill children who receive fentanyl by continuous infusion. Crit Care Med 1994;22(5):763-7.

[3] Fonsmark L, Rasmussen YH, Carl P. Occurrence of withdrawal in critically ill sedated children. Crit Care Med 1999; 27(1):196-9.

[4] Dominguez KD, Lomako DM, Katz RW, Kelly HW. Opioid withdrawal in critically ill neonates. Ann Pharmacother 2003;37(4):473-7.

[5] Ista E, van Dijk M, Gamel C, Tibboel D, de Hoog M. Withdrawal symptoms in critically ill children after long-term administration of sedatives and/or analgesics: a first evaluation. Crit Care Med 2008;36(8):2427-32.

[6] Anand KJ, Willson DF, Berger J, Harrison R, Meert KL, Zimmerman J, et al. Eunice Kennedy Shriver National Institute of Child Health and Human Development Collaborative Pediatric Critical Care Research Network. Tolerance and withdrawal from prolonged opioid use in critically ill children. Pediatrics 2010;125(5):e1208-25.

[7] Johnson PN, Harrison D, Allen C. Utility of transdermal fentanyl for prevention of iatrogenic opioid abstinence syndrome in children. J Opioid Manag 2010;6(2):117-24.

[8] Honey BL, Benefield RJ, Miller JL, Johnson PN. Alpha2receptor agonists for treatment and prevention of iatrogenic opioid abstinence syndrome in critically ill patients. Ann Pharmacother 2009;43(9):1506-11.

[9] Lugo RA, MacLaren R, Cash J, Pribble CG, Vernon DD. Enteral methadone to expedite fentanyl discontinuation and prevent opioid abstinence syndrome in the PICU. Pharmacotherapy 2001;21(12):1566-73.

[10] Siddappa R, Fletcher JE, Heard AM, Kielma D, Cimino M, Heard CM. Methadone dosage for prevention of opioid withdrawal in children. Paediatr Anaesth 2003;13(9):805-10.

[11] Meyer MM, Berens RJ. Efficacy of an enteral 10-day methadone wean to prevent opioid withdrawal in fentanyl-tolerant pediatric intensive care unit patients. Pediatr Crit Care Med 2001;2(4):329-33.

[12] Berens RJ, Meyer MT, Mikhailov TA, Colpaert KD, Czarnecki ML, Ghanayem NS, et al. A prospective evaluation of opioid weaning in opioid-dependent pediatric critical care patients. Anesth Analg 2006;102(4):1045-50.

[13] Robertson RC, Darsey E, Fortenberry JD, Pettignano R, Hartley G. Evaluation of an opiate-weaning protocol using methadone in pediatric intensive care unit patients. Pediatr Crit Care Med 2000;1(2):119-23.

[14] Tobias JD, Deshpande JK, Gregory DF. Outpatient therapy of iatrogenic drug dependency following prolonged sedation in the pediatric intensive care unit. Intensive Care Med 1994;20(7):504-7.

[15] Tobias JD, Schleien CL, Haun SE. Methadone as treatment for iatrogenic narcotic dependency in pediatric intensive care unit patients. Crit Care Med 1990;18(11):1292-3.

[16] Tobias JD. Out-patient therapy of iatrogenic opioid dependency following prolonged sedation in the pediatric intensive care unit. J Intensive Care Med 1996;11(5):284-7.

[17] Buck Ml. Managing iatrogenic opioid dependence with methadone [Internet]. 2000 [updated 2000 July; cited 2010 Nov 9]. Available from: http://www.healthsystem.virginia. edu/alive/pediatrics/PharmNews/200007.pdf.

[18] Stata statistical software [Computer program]. Version 10. College Station, TX: StataCorp LP; 2007.

[19] Tobias JD. Tolerance, withdrawal, and physical dependency after long-term sedation and analgesia of children in the pediatric intensive care unit. Crit Care Med 2000;28(6): 2122-32.

[20] Anand KJ, Arnold JH. Opioid tolerance and dependence in infants and children. Crit Care Med 1994;22(2):334-42.

[21] Hughes J, Gill A, Leach HJ, Nunn AJ, Billingham I, Ratcliffe J, et. al. A prospective study of the adverse effects of midazolam on withdrawal in critically ill children. Acta Paediatr 1994;83(11):1194-9.

[22] Dominguez KD, Crowley MR, Coleman DM, Katz RW, Wilkins DG, Kelly HW. Withdrawal from lorazepam in critically ill children. Ann Pharmacother 2006;40(6):1035-9.

[23] van Engelen BG, Gimbrere JS, Booy LH. Benzodiazepine withdrawal reaction in two children following discontinuation of sedation with midazolam. Ann Pharmacother 1993; 27(5):579-81.

[24] Katz R, Kelly HW. Pharmacokinetics of continuous infusions of fentanyl in critically ill children. Crit Care Med 1993; 21(7):995-1000.

[25] Berde CB, Sethna NF, Holzman RS, Reidy RN, Gondek EJ. Pharmacokinetics of methadone in children and adolescents in the perioperative period [Abstract]. Anesthesiology 1987; 67(3):A519. 